\title{
Anesthetic Management of Patients Undergoing Renal Transplantation: A Review of A Two-year Experience
}

\author{
Burhan Dost ${ }^{1, \star}$, Muzeyyen Beldagli ${ }^{1}$, Cengiz Kaya ${ }^{1}$, Yasemin Burcu Ustun ${ }^{1}$, \\ Sezgin Bilgin ${ }^{1}$, Ersin Koksal ${ }^{1}$, Yakup Bostancl ${ }^{2}$
}

\author{
${ }^{1}$ Department of Anesthesiology and \\ Reanimation, Faculty of Medicine, \\ Ondokuz Mayis University, Samsun, \\ Turkey \\ ${ }^{2}$ Department of Urology, Faculty of \\ Medicine, Ondokuz Mayis University, \\ Samsun, Turkey
}

\section{*Correspondence}

burhandost@hotmail.com

(Burhan Dost)

\begin{abstract}
Objectives: This study reviewed the anesthetic management procedures used for renal transplantation patients at a university hospital in Turkey over a two-year period. Areas of interest included preoperative status, fluid management, perioperative complications, and postoperative analgesia. Methods: A retrospective review of hospital records and information collected from all patients undergoing renal transplantation, performed at Ondokuz Mayıs University Hospital from 2018 to 2020, was conducted. Results: A total of 90 renal transplants were performed during the study time period. Of the patients undergoing renal transplantation, $44.4 \%$ were female and $55.6 \%$ were male. 52 patients were recipients of cadaveric transplants and 38 patients received kidneys from living, related donors. The duration of surgery and cold ischemic time were longer in the recipients of the cadaveric transplants $(p<0.05)$. Delayed graft function was statistically significantly higher in the kidney transplants obtained from the cadaveric donors than the living donors $(p<0.05)$. Conclusions: This study showed that anesthesia for renal transplantation is a safe and effective method if a selected range of drugs and techniques is used. Applying a well-planned standard anesthesia protocol and updating it periodically in line with the current literature will contribute to improving the quality of the outcomes renal transplantation surgery.
\end{abstract}

\section{Keywords}

Kidney transplantation, Anesthesia management, Delayed graft function, Analgesia, Hemodynamics

\section{Introduction}

Renal transplantation is one of the treatment methods that provides positive outcomes in the treatment of patients with end-stage renal disease (ESRD) undergoing hemodialysis [1, 2]. In comparison to dialysis, successful transplantation reduces mortality by $50 \%$ in the majority of the patients, and it improves the quality of life [3]. Today, advancements in surgical techniques and post-transplantation care and the use of immunosuppressive medications have significantly improved renal transplantation outcomes $[4,5]$. The number of transplantations using grafts obtained from living donors has gradually increased in recent years due to the shortage of cadaveric organ donors. The conditions for patients undergoing surgery are more ideal since living donor kidney transplants are planned electively in comparison to cadaveric donor kidney transplants. A short cold ischemia time (CIT) has a positive impact on both prognosis and graft function $[6,7]$. Cardiovascular diseases, which are the major factors of morbidity and mortality in kidney transplant candidates, are also the most common cause of death post-transplantation [8]. While hypertension is observed in $90 \%$ of the patients with a glomerular filtration rate of less than $30 \%$, the rate of observing a coronary artery disease in ESRD is $25 \%$ [9]. The presence of cardiovascular diseases accompanied by the presence of metabolic and respiratory systemic diseases, in addition to the acid-base and electrolyte abnormalities due to uremia increase the importance of detailed preoperative evaluation.

This study aimed to report on our two years of experience in renal transplant surgery, which could help improve the quality of the transplantation process and optimize the anesthesia approach that is used in these procedures.

\section{Methods}

This retrospective analytical observational study was approved by the Clinical Trials Ethics Committee of Ondokuz Mayis University (Approval number: 2020/175).

\subsection{Study population}

The preoperative and intraoperative anesthesia records of 90 patients, who were admitted to Ondokuz May1s University Hospital between January 1, 2018 and January 1, 2020, were retrospectively evaluated. Patients aged 18 years and over, 
TA B L E 1. Demographic Data.

\begin{tabular}{|llc|}
\hline & Frequency n (\%) & \\
\hline Gender & Female/Male & $40(44.4) / 50(55.6)$ \\
\hline Type of surgery & Open/Laparoscopic & $78(86.7) / 12(13.3)$ \\
\hline Donor & Cadaver/Living & $52(57.8) / 38(42.2)$ \\
\hline Comorbidities & Hypertension & $54(60)$ \\
\hline & Diabetes mellitus & $10(11.1)$ \\
\hline Coronary artery disease & $8(8.9)$ \\
\hline Etiology of ESRD & Asthma & $3(3.3)$ \\
\hline & Goiter & $4(4.4)$ \\
\hline Hypertension & $32(35.6)$ \\
\hline Intraoperative period & Diabetes mellitus & $30(33.3)$ \\
\hline Postoperative complication & Nausea and vomiting & $7(7.7)$ \\
\hline & Graft nephrectomy & $5(5.6)$ \\
\hline & Other & $16(17.7)$ \\
\hline & Hemodialysis & $77(85.6)$ \\
\hline & Antihypertensives & $7(7.8)$ \\
\hline & $1(1.1)$ \\
\hline & $22(24.4)$ \\
\hline
\end{tabular}

who underwent renal transplantation with grafts obtained from living and cadaveric donors, were included in the study. Patients whose anesthesia registration forms and clinical data registry could not be accessed were excluded from the study.

\subsection{Data collection}

The following data were collected for each recipient: demographic characteristics (age, sex, etc.), medical comorbidities (diabetes mellitus, hypertension, asthma, coronary artery disease $[\mathrm{CAD}]$ ), history of dialysis, transplant data (including donor type and CIT), anesthesia type, drugs used in anesthesia management, surgery type (open/laparoscopic), vasopressor use, fluid type and volume, delayed graft function (DGF); which requires dialysis in the first week following a kidney transplant, and transfused blood products.

\subsection{Anesthetic management}

In our clinical practice, in the preoperative period before the induction of anesthesia, clear fluids are allowed for up to two hours and solids are allowed for up to 6 hours for fasting. As premedication, $300 \mathrm{mg}$ per oral (PO) ranitidine is given the night before surgery and $10 \mathrm{mg}$ IV metoclopramide and 150 mg PO ranitidine are given an hour before surgery. For nausea and vomiting prophylaxis, $4 \mathrm{mg}$ IV dexamethasone is administered 90 minutes before the induction of anesthesia (except for diabetic patients on insulin) and $4 \mathrm{mg}$ IV ondansetron is administered 30 minutes before the surgery is completed. After the patients were taken to the operation room, standard ASA (American Society of Anesthesiologists) monitors (electrocardiography, noninvasive arterial pressure, and peripheral oxy- gen saturation), invasive blood pressure from the right radial artery, and central venous pressure (CVP) monitoring from the right jugular vein were performed. All patients were sedated with $0.03 \mathrm{mg} / \mathrm{kg}$ midazolam and 0.01 to $0.2 \mathrm{mcg} / \mathrm{kg} / \mathrm{min}$ remifentanil infusion. Subsequently, induction with propofol 1 - $2 \mathrm{mg} / \mathrm{kg} /$ Ideal Body Weight (IBW), remifentanil infusion was initiated before induction $(0.5-1 \mathrm{mcg} / \mathrm{kg} / \mathrm{Lean}$ Body Weight [LBW], bolus in 30 - 60 seconds) and it continued (0.015 - 1 $\mathrm{mcg} / \mathrm{kg} / \mathrm{LBW} / \mathrm{min}$ ) throughout the surgery. For muscle relaxation $1 \mathrm{mg} / \mathrm{kg} / \mathrm{LBW}$ rocuronium was performed. After rocuronium was administered, intubation was performed when the train-of-four count and the post-tetanic count (PTC) were both zero, and intermittent bolus injections were made so that the PTC was zero. Halogenated inhalation anesthetics (desflurane and sevoflurane; 1 minimum alveolar concentration, $\mathrm{O}_{2}$ /air [fraction of inspired oxygen of 0.40], the inspiratory fresh gas flow of $2 \mathrm{~L} / \mathrm{min}$ ) were preferred in anesthesia maintenance. The effects of the neuromuscular block were antagonized using neostigmine at the end of the operation. In the postoperative period, pain management was provided by using $1 \mathrm{mg} / \mathrm{kg}$ IV tramadol and 1 gr IV paracetamol (every 8 hours). Numeric rating scale (NRS) pain score was recorded from 15th minute in recovery room followed by 1.-3.-6.-12.-18.-24. hours with two different conditions which are at rest and while coughing. When NRS score became $\geq 4$ Morphine 1 - 3 mg was applied.

\subsection{Surgical technique}

On the day of surgery, preoperative dialysis was performed on patients receiving dialysis treatment. The graft was placed in the right iliac fossa of the kidney when no contraindications 
TA B L E 2. Anesthetics Used for induction and Maintenance of Anesthesia.

\begin{tabular}{llc}
\multicolumn{2}{c}{ Frequency n (\%) } \\
Hypnotic & Propofol & $90(100)$ \\
\hline Neuromuscular Blockers & Rocuronium & $90(100)$ \\
\hline Inhalation agents & Sevoflurane & $44(48.9)$ \\
& Desflurane & $46(51.1)$ \\
\hline Analgesics & Paracetamol/Tramadol/ Morphine & $42(46.7) / 74(82.2) / 12(13.3)$ \\
Reverse & Neostigmine/None & $86(95.5) / 4(4.4)$ \\
\hline
\end{tabular}

TA B L E 3. Comparisons by Donor Type.

\begin{tabular}{|lccc|}
\hline Variables & Cadaver & Living & p \\
\hline Age & $43.3 \pm 10.8$ & $44.2 \pm 14.1$ & 0.467 \\
\hline Amount of Bleeding $(\mathrm{mL})$ & $196 \pm 129$ & $183 \pm 76.1$ & 0.473 \\
\hline Ringer's Lactate $(\mathrm{mL})$ & $1710 \pm 1164$ & $1316 \pm 1075$ & 0.079 \\
\hline Physiologic Saline Solution $(\mathrm{mL})$ & $1460 \pm 1104$ & $1570 \pm 1127$ & 0.76 \\
\hline & Median (minimum - maximum) & \\
\hline Duration of Surgery (min) & $210(155-480)$ & $190.5(140-300)$ & $\mathbf{0 . 0 0 1 *}$ \\
\hline Cold Ischemia Time (min) & $790(292-1440)$ & $121(68-274)$ & $\mathbf{0 . 0 0 0 1 *}$ \\
\hline & Frequency n (\%) & \\
\hline Delayed graft function (+/-) & $12(25) / 40(75)$ & $2(2.2) / 36(97.8)$ & $\mathbf{0 . 0 1 8 *}$ \\
\hline
\end{tabular}

a. Data are presented as Mean $\pm S D$, median (minimum - maximum) or number (\%).

$b .{ }^{*} p<0.05$ statistically significant versus living donor type group.

were observed. In vascular anastomosis, the renal artery was anastomosed end-to-side to the external iliac artery, and the renal vein was anastomosed to the external iliac vein end-toside. In laparoscopic kidney transplantation, after the right iliac fossa was explored, right external iliac vein and artery were freed from surrounding tissues. The kidney was placed into the abdomen through an incision of approximately $8 \mathrm{~cm}$. in the left lower quadrant of the abdomen. Next, the graft artery and vein were anastomosed to the external iliac artery and vein in a way similar to the open technique. Afterward, the graft kidney was perfused by opening vascular clamps. The ureteroneocystostomy was performed with the Lich-Gregoir technique using a double-j stent.

\subsection{Statistics}

After the data were collected, a database was created with the variables that were captured to determine their veracity. All the statistical analyses were performed using SPSS Version 22 software (IBM, Armonk, NY, USA). Conformity of quantitative data to normal distribution was examined by using Kolmogorov-Smirnov test. To compare the qualitative and quantitative variables, the Chi-square analysis and a Student's t-test, with a normal distribution, and the Mann-Whitney U test, without normal distribution, were used, respectively. The quantitative variables were expressed as mean \pm standard deviation (SD); the categorical variables were expressed as numbers (n) and percentages (\%). A p-value of $<0.05$ was considered statistically significant, and the results were evaluated at a $95 \%$ confidence interval (CI).

\section{Results}

Fifty-two patients received kidneys from cadaveric donors and 38 patients received kidneys from living donors. The demographic data of the patients are summarized in Table 1. The transplant surgeries were performed under general anesthesia. The drugs used for anesthesia management are shown in Table 2. None of the patients developed anesthesiarelated complications during the perioperative period.

The duration of surgery and CIT were longer in the cadaveric donor group than the living donor group ( $\mathrm{p}<0.05)$. DGF was statistically significantly higher in the kidney transplants obtained from the cadaveric donors than the living donors ( $p$ $<0.05)$. Although the mean amount of fluid administered intraoperatively was higher in the cadaveric donor group, no statistically significant difference was observed between the groups $(\mathrm{p}>0.05)$. Table 3 presents the comparisons by donor type.

\section{Discussion}

A total of 90 patients underwent renal transplantation (57.8\% from a cadaveric donor and $42.2 \%$ from a living donor) in our clinic from 2018 to 2020. In the United States (US), transplantation from a cadaveric or living donor is $70 \%$ and $30 \%$, respectively [10]. In comparison to transplantation from a cadaveric donor, transplantation from a living donor has several advantages: the procedure can be scheduled, the clinical status of the recipient is optimized, waiting times and CIT 
are decreased, as is the need for dialysis, and the prognosis and function of the graft are improved [11]. In our study, the duration of surgery and CIT were longer in the recipients of cadaveric transplants $(\mathrm{p}<0.05)$.

The average age of the patients is $43.3 \pm 10.8$ in patients with cadaver renal transplantation and $44.2 \pm 14.1$ in living donors. Kidney transplantation is the preferred treatment method in patients with end-stage renal disease, due to both survival advantage and the increase it provides in the quality of life. Due to the adverse effects of prolonged dialysis treatments on organ transplantation, the inclination to perform the transplantation in the earliest stage possible has been getting stronger around the world in recent years [12]. In our series, $14.4 \%$ of the patients underwent kidney transplantation in the early stage, before dialysis treatment was initiated.

Because the mortality rates are lower among patients that need renal replacement therapy and that have undergone renal transplantation, this treatment represents an alternative method [13]. Ensuring perioperative hemodynamic stability is the factor that directly affects function and mortality after transplantation. However, to date, no previous study has provided sufficient evidence for the ideal anesthetic technique. Cardiovascular diseases are the most important cause of mortality in patients awaiting renal transplantation [14]. Hypertension and CAD are common complications among patients with ESRD [15]. Compatible with the data reported in the literature, in the present study, the number of patients with hypertension and CAD were also high (Table 1).

Due to concerns about coagulopathy, general anesthesia is preferred over regional anesthesia in patients that undergo transplantation surgery. General anesthesia is induced with either intravenous drugs or volatile agents, whereas volatile anesthetics are frequently used for maintenance [16]. Propofol is a short-acting and highly lipophilic intravenous anesthetic that is primarily metabolized in the liver. It is used as the first-line agent in induction since its pharmacokinetics and dynamics are largely preserved in patients with ESRD [17, 18]. Consequently, in the present study, anesthesia was induced using propofol in all patients. Patients with ESRD mostly suffer from hypovolemia and coexisting cardiac problems. Therefore, the titration of the propofol dose is extremely important for maintaining hemodynamic stability in this patient group. Furthermore, all drugs differ in their distribution volume and plasma protein binding capacity, making optimal titration of drugs very important [19]. In the present study, the remifentanil infusion was initiated before induction, and it continued throughout the surgery. A smoother induction was achieved by suppressing the sympathetic response that occurred after laryngoscopy and endotracheal intubation by initiating the use of remifentanil before induction [20]. Remifentanil, an opioid with an ultrashort-acting and highly lipophilic profile, was preferred as an analgesic in the present study because dose adjustment is not required since its pharmacokinetics are unchanged even in patients with ESRD. Furthermore, remifentanil has a very short terminal half-life (10 minutes) because it is rapidly metabolized by plasma and tissue esterases. No clinically significant accumulation of metabolites is observed with its infusion. It has the shortest context-sensitive half-life among the opioids, allowing it to be titrated easily, thereby avoiding cardiovascular adverse effects [21, 22].

In the present study, halogenated inhalation anesthetics (desflurane and sevoflurane) were preferred since elimination predominantly occurs through exhalation (independently from kidney function) in anesthesia maintenance. Thanks to their low solubility in the blood, desflurane and sevoflurane provide rapid and consistent recovery, and they do not cause hemodynamic instability. Some studies have reported that sevoflurane can be used safely in patients with ESRD, although there are concerns regarding compound A formation and renal toxicity $[23,24]$.

Non-depolarizing muscle relaxants are polarized drugs with a hydrophilic structure. Cisatracurium and atracurium can be safely used in patients with ESRD since they are metabolized by non-specific plasma esterases and through non-enzymatic pathways (Hofmann elimination). Since there were problems regarding the supply of these agents in our clinic, rocuronium was used, which was thought to be a good alternative. Rocuronium is a muscle relaxant with a low volume of distribution and no active metabolites, making it a good alternative in transplant patients [25]. In the present study, the effects of neuromuscular blockade were reversed using neostigmine, an inhibitor of acetylcholinesterase. Sugammadex is an effective reversal agent; it permanently creates a complex, excreted through kidneys, with rocuronium, which blocks its effect. Furthermore, sugammadex does not cause the side effects caused by acetylcholinesterase inhibitors, it is superior in preventing a postoperative residual block, and it provides rapid neuromuscular recovery. Thus, it is widely used in clinical practice [26]. However, in the present study, sugammadex could not be used in any patient because the manufacturer did not recommend using it if the creatinine clearance value was $<$ $30 \mathrm{~mL} / \mathrm{min}$. On the other hand, there are studies in the literature showing that it can be used safely and effectively in patients with severe renal / liver failure [27, 28].

In renal transplant patients, fluid management is critically important in terms of clinical outcomes. Crystalloids are the first choice in the intraoperative fluid regimen. Fluids containing potassium should be avoided [29]. Considering the fluids used in the intraoperative period, crystalloids were used in all patients (mean: $3 \mathrm{~L}$ ) in the present study. Colloids are often used as volume expanders; however, their superiority to crystalloids has not been reported yet [30]. Perioperative fluids should maintain adequate intravascular volume and ensure adequate perfusion in the transplanted kidney. These patients can easily experience hypovolemic shock with dialysis, and autoregulation of the transplanted denervated kidney graft has changed. Optimal intraoperative fluid management is essential for preventing DGF; it affects approximately one-quarter of cadaveric donor kidney transplant recipients. In the present study, DGF was statistically significantly higher in the kidney transplants obtained from the cadaveric donors than the living donors $(p=0.018)$. Acute tubular necrosis (ATN) is one of the common causes of DGF. Intraoperative inadequate organ perfusion, as well as surgical factors such as long CIT and anastomosis time, contributes to the development of ATN. Aggressive fluid therapy $(30 \mathrm{~mL} / \mathrm{kg})$ is generally recommended for renal transplant patients, except those with heart disease. Central venous pressure (CVP) monitoring and aggressive 
fluid regimen are applied in accordance with the traditional method. In our clinic, the initial CVP value and comorbidities are taken as the point of origin for determining fluid therapy, and CVP is maintained at $12-15 \mathrm{mmHg}$, not exceeding 30 $\mathrm{mL} / \mathrm{kg}$ of fluid. However, Gasperi et al. [31] reported that they successfully applied restrictive fluid therapy $(15 \mathrm{~mL} / \mathrm{kg})$ in eligible patients. A recent study comparing both fluid therapies reported that the restrictive fluid regimen significantly reduced the incidence of postoperative DGF and ileus [32]. Rather than strictly adhering to protocols, performing an individualized fluid therapy is recommended by evaluating the patient's fluid status at the beginning of the intraoperative period; the use of a restrictive fluid regimen is recommended, particularly in patients with poor myocardial function. In the present study, no patient had a CVP value $<10 \mathrm{mmHg}$ and no patient received $15 \mathrm{~mL} / \mathrm{kg}$ fluid therapy. It would be a rational approach to apply restrictive or even targeted fluid therapy by using non-invasive monitoring techniques in long-term surgeries [33]. In future follow-ups, we aim to apply restrictive fluid treatment protocols in selected patients with advanced monitoring techniques.

Blood transfusion should be avoided in the intraoperative period, if possible. However, blood transfusion should be performed particularly in patients with ongoing surgical bleeding and a hemoglobin value ranging from $<7 \mathrm{~g} / \mathrm{dL}$ to $8 \mathrm{~g} / \mathrm{dL}$. Anemia is common in patients with ESRD [34]. Similarly, in the present study, most of the patients had anemia [35]. However, blood transfusion was performed in only seven (7.8\%) patients.

Patients undergoing renal transplantation typically have mild-to-moderate postoperative pain [36]. Epidural anesthesia can be administered in these patients; however, its use is limited due to the hemodynamic effects and changes in coagulation. Regional methods, such as a transversus abdominis plane block, can be added to multimodal analgesia [37]. Similar to patients with kidney dysfunction, nonsteroidal anti-inflammatory drugs (NSAIDs) are not recommended in transplant recipients in the multimodal approach. Normeperidine, the active metabolite of meperidine, is also not preferred since it causes respiratory depression and it has neuroexcitatory effects, such as seizure. The development of postoperative respiratory depression should be carefully monitored since the pharmacokinetics of remifentanil, fentanyl, and sufentanil are heterogeneous in these patients [38]. In the present study, NSAIDs were observed not to be preferred, paracetamol and tramadol were generally used for postoperative analgesia, and the rate of morphine use was lower. Morphine was observed to be most preferred drug in patients receiving transplants from living donors. This may be due to the fact that CIT, duration of surgery, and anesthesia are shorter in living donors and postoperative DGF development is expected to be less. In the postoperative period, analgesia was induced using tramadol.

\section{Conclusions}

Anesthetic management during renal transplantation mainly aims to maintain hemodynamic stability, which helps reduce the incidence of DGF and acute postoperative tubular necrosis to optimize renal graft function, thus improving the patient's quality of life. The anesthetic approach used in the present study prevented the development of serious postoperative complications, and it ensured a safe and stable perioperative period.

In conclusion, applying a well-planned standard anesthesia protocol and updating it periodically in line with the current literature will contribute to improving the quality of the outcomes renal transplantation surgery.

\section{ACKNOWLEDGMENTS}

Thanks to all the peer reviewers and editors for their opinions and suggestions.

\section{CONFLICTS OF INTEREST}

The authors declare that there is no conflict of interest regarding the publication of this article.

\section{DATA AVAILABILITY}

The data used to support the findings of this study are available from the corresponding author upon request.

\section{AUTHORS' CONTRIBUTIONS}

$\mathrm{BD}, \mathrm{CK}$ and BYU designed the research study. MB performed the research. SB provided help. YB analyzed the data. BD, $\mathrm{CK}, \mathrm{EK}$ and BYU wrote the manuscript.

\section{FUNDING INFORMATION}

This research received no specific grant from any funding agency in the public, commercial, or not-for-profit sectors. At the time of data acquisition, the authors were employed at Faculty of Medicine, Ondokuz Mayıs University, Samsun, Turkey, where the study was conducted.

\section{REFERENCES}

[1] Held PJ, McCormick F, Ojo A, et al. A Cost-Benefit Analysis of Government Compensation of Kidney Donors. Am J Transplant. 2016;16:877-885.

[2] Rocha MJ, Ferreira S, Martins LS, et al. Cost analysis of renal replacement therapy by transplant in a system of bundled payment of dialysis. Clin Transplant. 2012;26:529-531.

[3] Wolfe RA, Ashby VB, Milford EL, et al. Comparison of mortality in all patients on dialysis, patients on dialysis awaiting transplantation, and recipients of a first cadaveric transplant. N Engl J Med. 1999;341:1725e30.

[4] Steinman TI, Becker BN, Frost AE, et al. Guidelines for the referral and management of patients eligible for solid organ transplantation. Transplantation. 2001;71:1189-1204.

[5] Ramos EL, Kasiske BL, Alexander SR, et al. The evaluation of candidates for renal transplantation. The current practice of U.S. transplant centers. Transplantation. 1994;57:490-497.

[6] Nemati E, Einollahi B, Pezeshki ML, et al. Does kidney transplantation with deceased or living donor affect graft survival? Nephro Urol Mon. 2014;6:e12182.

[7] Pence HH, Sert ÖZ, Bozkurt H, et al. The Effects of Laparoscopic Colon Surgery on Postoperative Renal Functions. Bakırköy Tıp Dergisi. 2019;15:193-197. 
[8] Pilmore H, Dent H, Chang S, et al. Reduction in cardiovascular death after kidney transplantation. Transplantation. 2010;89:851e7.

[9] Schmid S, Jungwirth B. Anaesthesia for renal transplant surgery: an update. Eur J Anaesthesiol. 2012;29:552e8.

[10] Organ Procurement and Transplantation Network. Available at: https://optn.transplant.hrsa.gov/data/view-datareports/national-data/\#.

[11] E. Nemati, B. Einollahi, M.L. Pezeshki, et al. Does kidney transplantation with deceased or living donor affect graft survival? Nephrourol Mon. 2014;5:e12182.

[12] Liyanage T, Ninomiya T, Jha V, et al. Worldwide access to treatment for end-stage kidney disease: a systematic review. Lancet. 2015;385:19751982.

[13] Kaballo MA, Canney M, O'Kelly P, et al. A comparative analysis of survival of patients on dialysis and after kidney transplantation. Clin Kidney J. 2017;11:389e93.

[14] Pilmore H, Dent H, Chang S, et al. Reduction in cardiovascular death after kidney transplantation. Transplantation 2010;89:851e7.

[15] Schmid S, Jungwirth B. Anaesthesia for renal transplant surgery: an update. Eur J Anaesthesiol. 2012;29:552e8.

[16] Aniskevich S, Pai S-L, Shine TS. Anesthetic pharmacology for kidney transplantation. Curr Clin Pharmacol. 2015;10: 47e53.

[17] Kirvelä M, Olkkola KT, Rosenberg PH, et al. Pharmacokinetics of propofol and haemodynamic changes during induction of anaesthesia in uraemic patients. Br J Anaesth. 1992;68:178.

[18] Kar, S. K., Khurana, H. S., Ganguly, T. Anesthesia management of renal transplantation: an update. Anaesthesia, Pain \& Intensive Care. 2019;22:383-392.

[19] Aniskevich S, Pai S-L, Shine TS. Anesthetic pharmacology for kidney transplantation. Curr Clin Pharmacol. 2015;10: 47e53.

[20] Kar, S. K., Khurana, H. S., Ganguly, T. Anesthesia management of renal transplantation: an update. Anaesthesia, Pain \& Intensive Care, 2019;22:383-392.

[21] Lemmens H.J.M., Ingrande J. Anesthesia and Intraoperative Management of Renal Transplantation. Anesthesia and Perioperative Care for Organ Transplantation. Springer, New York, NY. 2017.

[22] Gyulai, F. (2018). End-Stage Renal Disease-Patient For Kidney Transplant Blood Products. In: Straker T, Rajan S, editors. Anesthesiology: A Problem-Based Learning Approach, Oxford University Press; p155-165.

[23] Nishiyama T, Aibiki M, Hanaoka K. Inorganic fluoride kinetics and renal tubular function after sevoflurane anesthesia in chronic renal failure patients receiving hemodialysis. Anesth Analg. 1996;83:574.

[24] Hendrikus J M Lemmens. Anesthesia for kidney transplantation. In: UpToDate, Daniel C Brennan, Michael F O’Connor (Ed), UpToDate, Waltham, MA, 2020.

[25] Wierda JM, Kleef UW, Lambalk LM, et al. The pharmacodynamics and pharmacokinetics of Org 9426, a new non-depolarizing neuromuscular blocking agent, in patients anaesthetized with nitrous oxide, halothane and fentanyl. Can J Anaesth. 1991;38:430-435.

[26] Jones PM, Turkstra TP. Urgent usage of sugammadex to treat residual neuromuscular blockade in the PACU. Anesth Analg. 2007;105:877-878.

[27] Adams DR, Tollinche LE, Yeoh CB, et al. Short-term safety and effectiveness of sugammadex for surgical patients with end-stage renal disease: a two-centre retrospective study. Anaesthesia. 2020;75:348-352.

[28] Deana, C., Barbariol, F., D'Incà, S. et al. SUGAMMADEX versus neostigmine after ROCURONIUM continuous infusion in patients undergoing liver transplantation. BMC Anesthesiol. 2020;20:70.

[29] Chaumont M, Racapé J, Broeders N, et al. Delayed Graft Function in Kidney Transplants: Time Evolution, Role of Acute Rejection, Risk Factors, and Impact on Patient and Graft Outcome. J Transplant. 2015;2015:163757.

[30] Hadimioglu N, Saadawy I, Saglam T, et al. The effect of different crystalloid solutions on acid-base balance and early kidney function after kidney transplantation. Anesth Analgesia. 2008;107:264e9.

[31] De Gasperi A, Narcisi S, Mazza E, et al. Perioperative fluid management in kidney transplantation: is volume overload still mandatory for graft function? Transplant Proc. 2006;38:807-809.

[32] Cavaleri M, Veroux M, Palermo F, et al. Perioperative Goal-Directed Therapy during Kidney Transplantation: An Impact Evaluation on the Major Postoperative Complications. J Clin Med. 2019;8:80.

[33] Della Rocca G, Vetrugno L, Tripi G, et al. Liberal or restricted fluid administration: are we ready for a proposal of a restricted intraoperative approach? BMC Anesthesiol. 2014;14:62.

[34] Hovaguimian F, Myles PS. Restrictive versus Liberal Transfusion Strategy in the Perioperative and Acute Care Settings: A Context-specific Systematic Review and Meta-analysis of Randomized Controlled Trials. Anesthesiology. 2016; 125:46.

[35] Makroo RN, Kakkar B, Chowdhry M, et al. Retrospective analysis of perioperative transfusion requirements in living donor renal transplantation. Transfus Apher Sci. 2016;54:405e9.

[36] Yost CS, Niemann CU. Anesthesia for Abdominal Organ Transplantation. In: Miller's Anesthesia. Philadelphia: Elsevier Churchill Livingstone; 2010. p. 2155-2184.

[37] Rohan, V.S., Taber, D.J., Patel, N., et al. Impact of a Multidisciplinary Multimodal Opioid Minimization Initiative in Kidney Transplant Recipients. Clin Transplant. 2020. doi:10.1111/ctr.14006.

[38] Koehntop DE, Rodman JH. Fentanyl pharmacokinetics in patients undergoing renal transplantation. Pharmacotherapy. 1997;17:746-752.

How to cite this article: Burhan Dost, Muzeyyen Beldagli, Cengiz Kaya, Yasemin Burcu Ustun, Sezgin Bilgin, Ersin Koksal, et al. Anesthetic Management of Patients Undergoing Renal Transplantation: A Review of A Two-year Experience. Signa Vitae. 2021;17(1):95-100. doi:10.22514/sv.2020.16.0078. 